\title{
Clostridium difficile infection at a geriatric acute- care hospital in Switzerland between 2008 and 2014: a retrospective cohort study
}

\author{
D Pires ${ }^{1 *}$, V Sauvan ${ }^{1}$, V Zanichelli ${ }^{1}$, V Prendki ${ }^{2}$, J Reny ${ }^{2}$, S Harbarth ${ }^{1,3}$, B Huttner ${ }^{1,3}$ \\ From 3rd International Conference on Prevention and Infection Control (ICPIC 2015) \\ Geneva, Switzerland. 16-19 June 2015
}

\section{Introduction}

Clostridium difficile infection (CDI) epidemiology has changed with an increase in incidence and severity, particularly among the elderly.

\section{Objectives}

We aim to describe CDI epidemiology and assess the effect of infection control measures in the geriatric hospital of Geneva University Hospitals.

\section{Methods}

Retrospective cohort study of CDI patients, identified via active surveillance of the infection control program, at a 300 bed acute-care geriatric hospital in 2008-2014. We analyzed CDI incidence, surveillance case definition (hospital-onset healthcare facility-associated, $\mathrm{HO}$ HCFA), recurrence, demographics, antibiotic exposure, time at risk and in-hospital mortality. Positive tests $\leq 14$ days of diagnosis were excluded. Cell cytotoxicity test was replaced by PCR detection of $C$. difficile toxin B in $01 / 2010$. Infection control measures included contact precautions, environmental cleaning and single room policy. Overall antibiotic use was also analyzed.

\section{Results}

We identified 231 CDI cases resulting in mean incidence of 92.1 cases/10000 admissions. 194 cases (83.9\%) were HO-HCFA. Mean age was $85(+-7.3)$ years; $26.4 \%$ $(\mathrm{n}=61)$ were male. Mean CDI incidence (cases/10000 admissions/year) was 124.7(2008), 82.9(2009), 107.9 (2010), 100.4(2011), 139.5(2012), 42.9(2013), 46.5(2014). Mean time at risk was 23.5 (IQR 6-34) days. 180

${ }^{1}$ Infection Control Unit, Geneva University Hospitals, Geneva, Switzerland Full list of author information is available at the end of the article patients $(77.9 \%)$ received any antibiotics and 59.3\% $(\mathrm{n}=137)$ "high-risk" antibiotics in the previous month. 46 patients (29.9\%) had a recurrence at a mean of 25 days. In-hospital mortality was $34.4 \%(\mathrm{n}=76)$. From $01 / 2011$ to $03 / 2013$ single-room policy for CDI cases was suspended. During this period CDI incidence was 115.6 cases/10000 admissions, compared to 39.2 cases/ 10000 admissions after policy reinstitution (IRR 3.02, 95\% CI 2.01-4.53). Overall antibiotic use slightly decreased from 260 DDD/1000PD in 2008 to 218 DDD/ 1000PD in 2013.

\section{Conclusion}

We observed a low incidence of CDI at the acute care geriatric hospital after a peak in 2012. The decreasing trend is likely the result of multifaceted measures such as improved antibiotic use, improved environmental cleaning, hand hygiene promotion and reinstitution of a single-room policy.

\section{Disclosure of interest}

None declared.

\section{Authors' details}

${ }^{1}$ Infection Control Unit, Geneva University Hospitals, Geneva, Switzerland.

${ }^{2}$ Division of Internal Medicine and Rehabilitation, Geneva University Hospitals, Geneva, Switzerland. ํivision of Infectious Diseases, Geneva University Hospitals, Geneva, Switzerland.

Published: 16 June 2015

doi:10.1186/2047-2994-4-S1-035

Cite this article as: Pires et al:: Clostridium difficile infection at a geriatric acute-care hospital in Switzerland between 2008 and 2014: a retrospective cohort study. Antimicrobial Resistance and Infection Control 2015 4(Suppl 1):035. 